\title{
Hyperimmunoglobulin E Syndrome-like Symptoms in a Hemodialysis Patient who Developed Multiple Subcutaneous Staphylococcus aureus Abscesses
}

\author{
Kazuki Yokota, Toshikazu Kanemitsu and Naoya Igaki
}

\begin{abstract}
Hyperimmunoglobulin E syndrome (HIES) is sporadically triggered by de novo genetic mutations of the signal transducer and activator of transcription 3 (STAT3). Dialysis patients frequently exhibit HIES-like symptoms including elevated IgE levels and recurrent bacterial infections. We herein report the case of a 74year-old man on hemodialysis (HD) who developed marked $\mathrm{IgE}$ elevation and multiple subcutaneous cold Staphylococcus aureus abscesses. We are the first to report that a dialysis patient presented with HIES-like symptoms without the characteristic facies or bone and connective tissue abnormalities. A STAT3 gene analysis was conducted as part of the differential diagnosis.
\end{abstract}

Key words: hyperimmunoglobulin E syndrome (HIES), signal transducer and activator of transcription 3 (STAT3), dialysis patients

(Intern Med 52: 2367-2370, 2013)

(DOI: 10.2169/internalmedicine.52.0751)

\section{Introduction}

Hyperimmunoglobulin E syndrome (HIES) is a primary immunodeficiency that manifests with recurrent infections, particularly by Staphylococcus aureus, and is characterized by distinctive facies, joint hyperextensibility, multiple bone fractures, scoliosis and delayed shedding of the primary teeth. It was recently reported that HIES is sporadically triggered by de novo genetic mutations of signal transducer and activator of transcription 3 [STAT3 (1)]. Dialysis patients also frequently exhibit elevated IgE levels for various reasons and suffer from recurrent bacterial infections. In medical examinations of dialysis patients, clinicians often encounter cases that include the differential diagnosis of HIES.

We herein report a case in which a hemodialysis (HD) patient developed HIES-like symptoms including marked elevation of the IgE levels and multiple Staphylococcus aureus subcutaneous abscesses, although a STAT3 gene analysis ruled out HIES.

\section{Case Report}

In May 2011, a 74-year-old Japanese man was admitted to our hospital with fatigue and 4- to 5-cm cold abscesses on his left elbow (Fig. 1A) and the back of his head. He had previously been treated for atopic dermatitis with a steroid ointment. HD had been initiated for diabetic renal failure in March 2011. On admission, the laboratory results were as follows: white blood cell (WBC): $10,850 / \mu \mathrm{L}$ (eosinophil count: 173/ $\mu \mathrm{L}$ ), C-reactive protein (CRP): 8.08 $\mathrm{mg} / \mathrm{dL}$ and IgE: 25,747 IU/mL (Table). By the following day, the cold abscesses had spread to the anterior surface of the patient's neck (Fig. 1B) and right lower abdomen (Fig. 1C). The blood cultures and parasitological examinations were negative (Table). The patient was treated with a course of antibiotics, and all of the abscesses were drained. The fluid from the abscesses was cultured, and the causative bacterium was identified as Staphylococcus aureus, which exhibited sensitivity to all the antibiotics tested. By day 33 after admission, the patient's inflammatory response had improved to a WBC count of $5,250 / \mu \mathrm{L}$ (eosinophil count: 
Table. Results of Laboratory Tests of the Patient

\begin{tabular}{|c|c|c|c|}
\hline \multicolumn{2}{|l|}{ Peripheral blood count } & \multicolumn{2}{|l|}{ Blood biochemistry } \\
\hline WBC & $10850 / \mu \mathrm{L}$ & $\mathrm{TP}$ & $5.9 \mathrm{~g} / \mathrm{dL}$ \\
\hline Neutrophil & $84.8 \%$ & Alb & $3.1 \mathrm{~g} / \mathrm{dL}$ \\
\hline Eosinophil & $1.6 \%$ & AST & $28 \mathrm{IU} / \mathrm{L}$ \\
\hline ( eosinophil count & $\left.173 / \mathrm{mm}^{3}\right)$ & ALT & $19 \mathrm{IU} / \mathrm{L}$ \\
\hline Lymphocyte & $8.2 \%$ & BUN & $56.8 \mathrm{mg} / \mathrm{dL}$ \\
\hline Monocyte & $5.1 \%$ & Cre & $8.73 \mathrm{mg} / \mathrm{dL}$ \\
\hline $\mathrm{RBC}$ & $256 \times 10^{4} / \mu \mathrm{L}$ & UA & $10.7 \mathrm{mg} / \mathrm{dL}$ \\
\hline $\mathrm{Hb}$ & $7.7 \mathrm{~g} / \mathrm{dL}$ & $\mathrm{Na}$ & $133 \mathrm{mEq} / \mathrm{L}$ \\
\hline $\mathrm{Ht}$ & $23.4 \%$ & $\mathrm{~K}$ & $5.59 \mathrm{mEq} / \mathrm{L}$ \\
\hline \multirow[t]{2}{*}{ Plt } & \multirow[t]{2}{*}{$36.7 \times 10^{4} / \mu \mathrm{L}$} & $\mathrm{Cl}$ & $93 \mathrm{mEq} / \mathrm{L}$ \\
\hline & & $\mathrm{Ca}$ & $8.1 \mathrm{mg} / \mathrm{dlL}$ \\
\hline \multicolumn{2}{|c|}{ Immunologic blood tests } & $\mathrm{P}$ & $4.3 \mathrm{mg} / \mathrm{dL}$ \\
\hline C-reactive protein & $8.08 \mathrm{mg} / \mathrm{dL}$ & PPG & $511 \mathrm{mg} / \mathrm{dL}$ \\
\hline IgG & $1523 \mathrm{mg} / \mathrm{dL}$ & GA & $33.3 \%$ \\
\hline $\operatorname{Ig} \mathrm{A}$ & $480 \mathrm{mg} / \mathrm{dL}$ & T-Chol & $126 \mathrm{mg} / \mathrm{dL}$ \\
\hline $\operatorname{IgM}$ & $52 \mathrm{mg} / \mathrm{dL}$ & HDL-Chol & $36.8 \mathrm{mg} / \mathrm{dL}$ \\
\hline $\operatorname{IgE}$ & $25747 \mathrm{IU} / \mathrm{mL}$ & TG & $115 \mathrm{mg} / \mathrm{dL}$ \\
\hline$\beta$-D-glucan & $5.06 \mathrm{pg} / \mathrm{mL}$ & LDL-Chol & $68 \mathrm{mg} / \mathrm{dL}$ \\
\hline \multicolumn{2}{|c|}{ Candida albicans antigen } & & \\
\hline \multicolumn{4}{|c|}{ Parasite IgG antibody screening test } \\
\hline \multicolumn{2}{|c|}{ Canine filariasis } & Paragonimus westermani & $(-)$ \\
\hline Toxocara canis & $(-)$ & Fasciola hepatica & $(-)$ \\
\hline Anisaxis & $(-)$ & Liver fluke & $(-)$ \\
\hline Gnathostoma & $(-)$ & Sparganosis mansoni & $(-)$ \\
\hline Strongyloides stercoralis & oralis & Cysticercosis cellulosae & $(-)$ \\
\hline
\end{tabular}

PPG: postprandial plasma glucose, GA: glycoalbumin

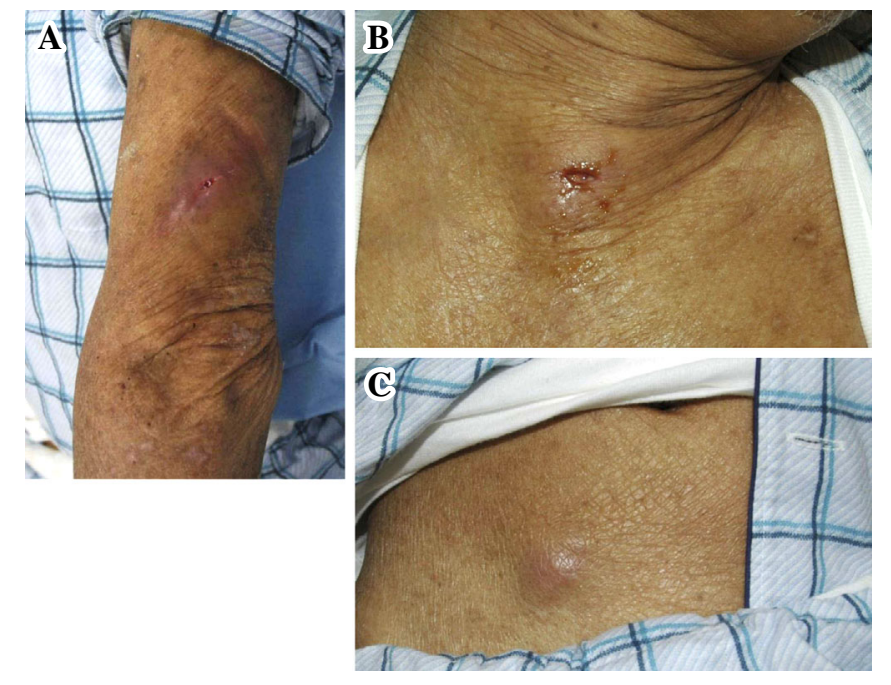

Figure 1. The patient was admitted to our hospital with fatigue and 4 to $5 \mathrm{~cm}$ cold abscesses on his left elbow (A) and the back of his head. By the following day, the cold abscesses had spread to the anterior suface of the patient's neck $(B)$ and right lower abdomen $(\mathrm{C})$.

$1,995 / \mu \mathrm{L}$ ) and a CRP level of $0.18 \mathrm{mg} / \mathrm{dL}$, and all of the abscesses had resolved (Fig. 2). However, the IgE value remained high $(18,535 \mathrm{IU} / \mathrm{mL})$.

Based on the presence of atopic dermatitis, multiple Staphylococcus aureus abscesses (possibly recurrent), marked IgE elevation and lack of a family history, a differ- ential diagnosis of sporadic type 1 HIES caused by de novo genetic mutations in STAT3 was made. After obtaining approval from the ethics committee of Takasago Municipal Hospital and informed consent from the patient, we performed a genetic analysis. Polymerase chain reaction (PCR) amplification of the patient's DNA was carried out using STAT3-specific primers, and a single-nucleotide polymorphism (SNP) analysis was conducted with a direct sequence using the PCR product as a template. Although exon 12 was not analyzed due to poor sequence quality, no mutations were found in the other STAT3 exons.

\section{Discussion}

Compared to the general population, dialysis patients more frequently suffer from eosinophilia and elevated IgE levels (2); however, the etiology of this phenomenon is unclear. Most physicians associate eosinophilia and elevated IgE levels with allergies or infection by parasites and fungal organisms. Bodner et al. suggested that eosinophilia in dialysis patients occurs due to the release of eosinophilotactic substances induced by the dialysis procedure itself (3). Some investigators have reported that eosinophilia may be induced in dialysis patients, not by renal disease, medications or the type of dialyzer or access, but by uremia or an allergic reaction to the dialysis equipment $(4,5)$.

In the present case, we initially hypothesized that the patient's eosinophilia and elevated IgE levels were due to 

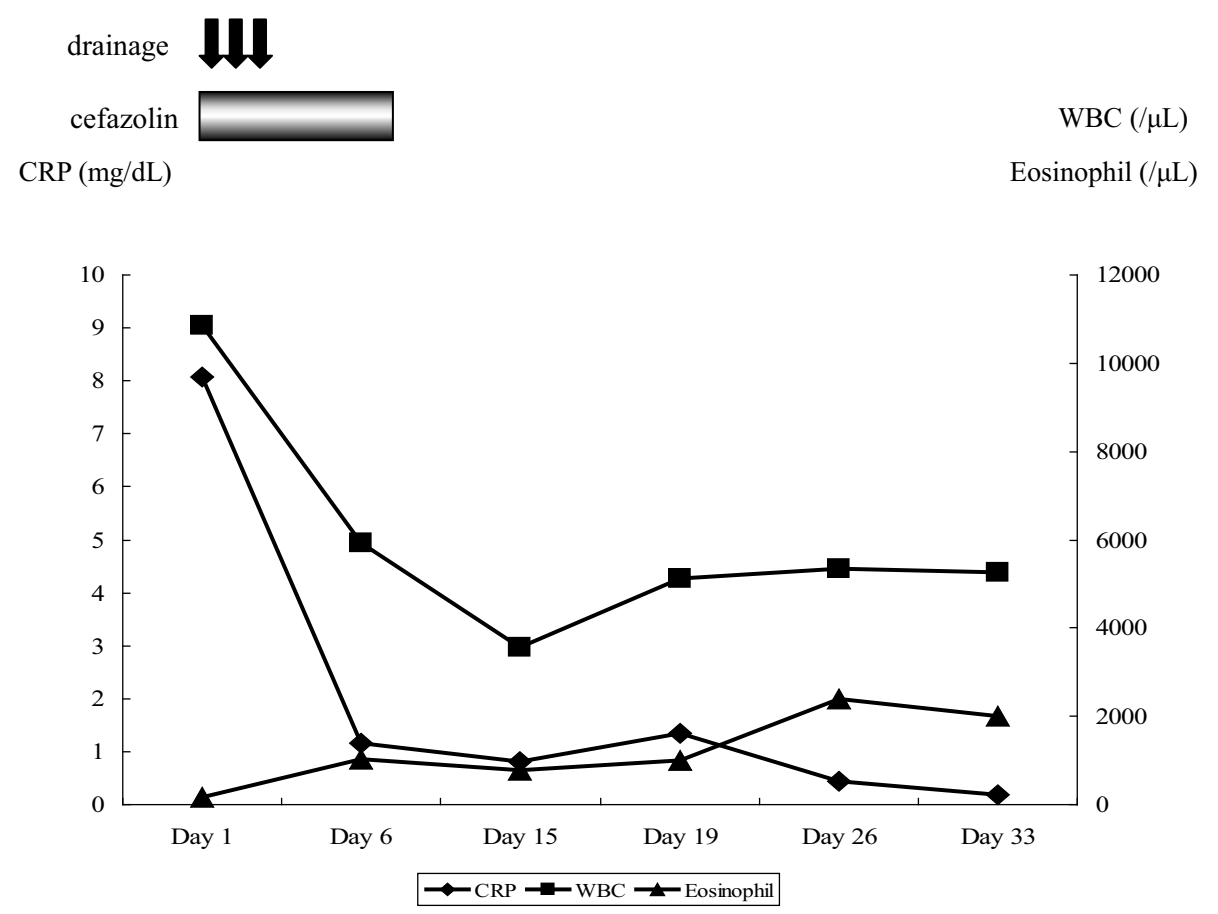

Figure 2. The patient was admitted to our hospital with fatigue and 4 to $5 \mathrm{~cm}$ cold abscesses on his left elbow and the back of his head. On admission, the laboratory results were as follows: WBC: 10,850/ $\mathrm{L}$ (eosinophil count: 173/ $\mu \mathrm{L}$ ) and CRP: $8.08 \mathrm{mg} / \mathrm{dL}$. On the following day, the cold abscesses had spread to the anterior surface of the patient's neck and right lower abdomen. He was treated with a course of antibiotics, and all of the abscesses were drained. On day 33 after admission, the patient's inflammatory reaction had improved to a WBC count of 5,250/ $\mu \mathrm{L}$ (eosinophil count: 1,995/ $\mu \mathrm{L}$ ) and a CRP level of $0.18 \mathrm{mg} / \mathrm{dL}$, and all of the abscesses had resolved.

atopic dermatitis that was worsened by uremia or an allergic reaction to the dialysis equipment on admission. However, we conducted a differential diagnosis because we observed the presence of multiple staphylococcal cold abscesses, which are characteristic of HIES. The blood cultures were negative in this case; however, methicillin-sensitive Staphylococcus aureus (MSSA) was identified in all of the subcutaneous abscesses. In HD patients, Staphylococcus aureus infection MSSA and even methicillin-resistant Staphylococcus aureus (MRSA) colonization are important clinical features (6). Staphylococcus aureus is the most common cause of dialysis-associated bacteremia and accounts for $25 \%$ of the bacteremia isolates obtained from such patients (7). In addition, Staphylococcus aureus infection can lead to various complications, such as infective endocarditis, osteomyelitis, abscess formation and septic arthritis (8). However, to the best of our knowledge, there are no reports in the literature of multiple subcutaneous cold abscesses occurring in a dialysis patient. Therefore, we sought to clinically distinguish this condition from HIES.

Patients with Type 1 HIES exhibit characteristic facies (including left-right facial asymmetry, a prominent forehead, depressed orbits, a wide nose bridge and an enlarged nose tip), joint hyperextensibility, multiple bone fractures, scoliosis and delayed shedding of the primary teeth; however, our patient exhibited no apparent abnormalities. A STAT3 gene analysis was required to precisely differentiate HIES in this case because some cases of HIES in patients without characteristic facies or bone and connective tissue abnormalities have been reported. In addition, eosinopenia has recently been reported to be a marker of sepsis (9), leading us to suspect that the current patient's low eosinophil count on admission may have been caused by a sepsis-like condition.

STAT3 mutations in HIES individuals preclude T-cell production of interleukin (IL)-17, which leads to impaired Thelper (Th) 17 cell differentiation (10). IL-17 production is required to protect against fungal infections and extracellular bacteria, as observed in mice with a targeted IL-17 mutation. Multiple HIES-like Staphylococcus aureus abscesses developed in this case; however, there were no apparent changes in the IL-17 levels due to the STAT3 mutations (the IL-17 levels were not measured). In this case, the results may be attributed to the patient's status as a compromised host, as he suffered from diabetes and was undergoing HD.

The genetic etiology of HIES was recently determined. While HIES was previously diagnosed based on clinical presentation, it can now be diagnosed at an early stage using gene analyses. STAT3 gene mutations have been identified in $70 \%$ of HIES individuals, and more than $90 \%$ of these STAT3 mutations are sporadic de novo mutations. However, the genetic basis for this condition in many HIES individuals remains to be completely elucidated. Additionally, in this case, a diagnosis of HIES could not be completely excluded because exon 12 was not analyzed due to poor sequence 
quality. Future research is needed to identify other genetic causes of HIES to increase the accuracy of gene analyses.

Eosinophilia and elevated IgE levels are commonly observed in dialysis patients. In addition, HIES-like Staphylococcus aureus infections develop easily in dialysis patients because the patients are compromised hosts. Therefore, dialysis patients may present with symptoms similar to those of HIES individuals. However, this is the first report in which a diagnosis of HIES was specifically excluded in a dialysis patient based on a STAT3 gene analysis. We are the first to report a STAT3 genetic analysis of a dialysis patient with HIES-like symptoms who lacked the characteristic facies and bone or connective tissues abnormalities. Naturally, a genetic analysis of STAT3 cannot be performed in all cases of elevated IgE levels and Staphylococcus aureus infections because HIES is a rare disease. Fortunately, we had the opportunity to conduct a STAT3 gene analysis and present the results of this case report.

The authors state that they have no Conflict of Interest (COI).

\section{References}

1. Minegishi Y, Karasuyama H. Genetic origins of hyper-IgE syndrome. Curr Allergy Asthma Rep 8: 386-391, 2008.
2. Barbee RA, Halonen M, Lebowitz M, Burrows B. Distribution of IgE in a community population sample: correlations with age, sex, and allergen skin test reactivity. Allergy Clini Immunol 68: 106$111,1981$.

3. Bodner G, Peer G, Zakuth V, Spirer ZH, Aviram A. Dialysisinduced eosinophilia. Nephron 32: 63-66, 1982.

4. Backenroth R, Spinowitz BS, Galler M, Golden RA, Rascoff JH, Charytan C. Comparison of eosinophilia in patients undergoing peritoneal dialysis and hemodialysis. Am J Kidney Dis 8: 186$191,1986$.

5. Gwinner W, Menne J, Lonnemann G, Bahlmann F, Koch KM, Haller H. Life-threatening complications of extracorporeal treatment in patients with severe eosinophilia. Int J Artif Organs 28: 1224-1227, 2005.

6. Lentino JR, Baddour LM, Wray M, Wong ES, Yu VL. Staphylococcus aureus and other bacteremias in hemodialysis patients: antibiotic therapy and surgical removal of access site. Infection $\mathbf{2 8}$ : 355-360, 2000.

7. Allon M. Dialysis catheter-related bacteremia: treatment and prophylaxis. Am J Kidney Dis 44: 779-791, 2004.

8. Nissenson AR, Dylan ML, Griffiths RI, et al. Clinical and economic outcomes of Staphylococcus aureus septicemia in ESRD patients receiving hemodialysis. Am J Kidney Dis 46: 301-308, 2005.

9. Abidi K, Khoudri I, Belayachi J, et al. Eosinopenia is a reliable marker of sepsis on admission to medical intensive care units. Criti Care 12: R59, 2008.

10. Milner JD, Brenchley JM, Laurence A, et al. Impaired $T_{H} 17$ cell differentiation in subjects with autosomal dominant hyper-IgE syndrome. Nature 452: 773-776, 2008.

(C) 2013 The Japanese Society of Internal Medicine http://www.naika.or.jp/imonline/index.html 